\title{
Lapurdum
}

Euskal ikerketen aldizkaria | Revue d'études basques |

Revista de estudios vascos | Basque studies review

$20 \mid 2017$

Numéro XX

\section{Paraboles, fables et fariboles : la découverte de l'occitan alpin au XIXe siècle}

\section{Philippe Martel}

\section{OpenEdition}

Journals

Édition électronique

URL : https://journals.openedition.org/lapurdum/3505

DOI : 10.4000/lapurdum.3505

ISSN : 1965-0655

Éditeur

IKER

Édition imprimée

Date de publication : 1 janvier 2017

Pagination : 103-114

ISBN : 978-2-95534-135-3

ISSN : 1273-3830

Référence électronique

Philippe Martel, «Paraboles, fables et fariboles : la découverte de l'occitan alpin au XIXe siècle », Lapurdum [En ligne], 20 | 2017, mis en ligne le 01 janvier 2021, consulté le 03 septembre 2021. URL : http://journals.openedition.org/lapurdum/3505; DOI : https://doi.org/10.4000/lapurdum.3505 


\section{Paraboles, fables et fariboles : la découverte de l'occitan alpin au XIXe siècle}

Philippe MARTEL

Université Paul-Valéry Montpellier 3

Comment visualiser simplement la variation des parlers locaux, avant l'invention des atlas linguistiques? Les premiers « dialectologues» du XIXe siècle ont une solution simple: juxtaposer des traductions d'un même texte dans plusieurs variantes, en laissant au lecteur le soin d'effectuer le travail de comparaison qui lui permettra de reconstituer un paysage. Avantage supplémentaire : une telle entreprise permet à la fois de mobiliser des correspondants nombreux un peu partout, et de fournir des témoignages écrits pour des parlers que nul poète n'a jusque là illustrés, sans avoir à attendre que des vocations d'auteur se manifestent, ou pas. On pense ici bien sûr à cette enquête Bourciez, lancée à la fin du XIXe siècle par le célèbre romaniste, et à laquelle Charles Videgain s'est intéressé pour ce qui concerne le domaine basque. Ou, pour l'est des Pyrénées, l'enquête Sacaze. Et, auparavant, il y a ces précurseurs que sont l'Adelung du Mithridates de 1806, à une échelle planétaire, cette fois (Courouau 2011, 79-101) puis, peu de temps après, l'enquête des deux Coquebert de Montbret, demeurée inédite dans l'ensemble, mais dont des extraits ont pu être publiés dès le XIXe siècle, et ont continué à l'être par la suite. Adelung avait choisi comme texte support le Pater. Coquebert, et nombre de ses successeurs ont préféré un texte tout aussi évangélique, mais plus long, la parabole de l'Enfant Prodigue : la faveur dont bénéficie ce texte ne s'est pas démentie, jusqu'à une époque récente, et il n'y a pas de raison de prédire que la mode passera. Mon propos ici n'est pas de traiter de ce sujet dans son ensemble, alors même que d'autres ont pu exploiter avec fruit les résultats de l'enquête impériale de 1807-1812, que ce soit sur la question des limites de l'occitan (G. Brun-Trigaud 1990) ou sur l'impact de l'enquête sur les débuts de la renaissance d'oc (René Merle 1990-2) Je me bornerai à un survol rapide des diverses enquêtes 
à base de traduction d'un texte-type qui ont concerné, plus tard dans le XIXe siècle, la partie nord-orientale de l'espace occitan, la zone alpine, entre vallée du Rhône et sources du Pô. En dehors du fait, anecdotique j'en conviens, que c'est la forme d'occitan que je connais le mieux, le choix de ce terrain me semble intéressant d'abord parce que plusieurs entreprises de traduction l'ont concerné au fil du XIXe siècle, et aussi et peut-être surtout parce que cet espace dialectal est traversé par une frontière nationale, celle qui sépare les Alpes méridionales françaises de leur versant piémontais (puis italien à partir de l'unification de la péninsule). On a donc là d'abord un corpus, mais aussi la possibilité de voir comment deux cultures nationales différentes, deux traditions philologiques aussi, ont pu se pencher sur un même ensemble linguistique, et comment elles ont répondu à un certain nombre de questions pratiques autant que théoriques : comment nommer ces parlers, comment les noter, quand n'existent encore ni l'alphabet phonétique Rousselot, ni l'API, comme enfin décrire leurs caractères principaux à partir de ce que révèle un texte somme toute assez bref.

Au total, nos corpus comparatifs nous fournissent aussi bien des photographies de l'état d'un parler à un moment donné que l'état des représentations qu'en ont ceux qui, sur ordre ou par goût, s'y sont intéressés.

\section{Collecter des parlers}

Au commencement, il y a donc la Parabole de l'Enfant prodigue, traduite de l'Evangile de Luc (15, 11-32). L'enquête Coquebert de Montbret fournit des spécimens représentatifs -une bonne trentaine -de la variation interne à chacun des départements de la zone occitane alpine (Drôme, Hautes Alpes, sud de l'Isère, nord des Basses Alpes et des Alpes Maritimes). Comme on sait, cette collection de paraboles est restée pour une bonne part inédite, et dispersée. Mais en 1831, Eugène Coquebert de Montbret en donne (sans signer) quelques-unes dans ses Mélanges sur les langues, dialectes et patois (Paris, Almanach du commerce). D’autres, ou les mêmes sont reprises quelques années plus tard par J. F. Schnackenburg dans son Tableau synoptique et comparatif des idiomes populaires ou patois de la France (Paris Berlin 1840), et, encore en 1882, mais à Niort cette fois, par Louis Favre (Les patois de la France). Il s'agit là de recueils concernant l'ensemble du territoire national (reste prudemment de côté ce qui concerne des régions perdues au traité de Vienne de 1815...). Mais sur place, d'autres, anciens préfets à qui la Restauration laisse des loisirs, ou érudits qui ont été associés à l'enquête, peuvent publier ce qu'ils ont en leur possession. Il existe donc un modèle, méthodologique et textuel, disponible pour d'autres, plus tard. Et d'autres, il va y en avoir.

Y compris de l'autre côté de la frontière franco-italienne, dans ces vallées occitanophones du Piémont que l'enquête impériale n'avait fait qu'effleurer -un texte de la val Pellice, «vaudoise » (protestante). En 1853, Bernardo Biondelli publie un Saggio sui dialetti gallo-italici : c'est la première étude sur les parlers du nord d'une Italie qui n'est pas encore unifiée mais se pose déjà la questione della lingua. Cet ouvrage assez épais entend décrire les dialectes piémontais, lombard, émilien, et est illustré de traductions de la parabole qu'il a demandées à des savants locaux (souvent des prêtres). Les parlers «alpigiani » du Piémont sont pris en compte, et Biondelli fournit donc une dizaine de spécimens venus de la plupart des vallées occitanophones (seules manquent celles de la Corsaglia, à l'extrême sud et celle de la Doire Riparia, au nord), plus deux spécimens du francoprovençal (non identifié comme tel bien sûr). 
En 1877 paraît le Patois des Alpes Cottiennes, dû à deux érudits haut-alpins (Chabrand-de Rochas 1877) On y trouve un lexique et des éléments de grammaire d'un parler du Queyras (celui d'Aiguilles à la base, semble-t-il). Et on y trouve la parabole, dans le parler décrit, en ancien vaudois et vaudois moderne (de Val Pellice là encore, mais dans une variante de la basse vallée teintée de piémontais, et fournie par le pasteur Pierre Bert qui avait déjà collaboré avec Biondelli), plus les parlers d'Oulx (vallée de la Doire, en amont de Suse, tout au nord des vallées occitanophones, de Pragelas (haut Chisone un peu au dessus de Fenestrelle), pour le côté italien, du Monêtier, d'Embrun et de Barcelonnette pour le versant français. Comme Biondelli, les deux auteurs ont fait appel à des traducteurs, le plus souvent anonymes, sans recourir au corpus de l'enquête de 1807-1812.

La parabole de Saint Luc n'est pas le seul support possible pour qui entend donner une idée de la diversité des parlers d'une région donnée. Pour la zone occitane alpine, il faut mentionner trois autres expériences, d'ampleur inégale, et qui, dans certains cas ont une visée à la fois informative (à quoi ressemblent les idiomes de notre zone) et «monumentale», si l'on peut dire : ouvrir au « patois», à travers la traduction d'un texte littéraire plus ou moins prestigieux, les portes d'un registre de la langue plus élevé que celui de la Parabole.

Certaines de ces entreprises n'envisagent qu'une partie de l'espace qui nous intéresse. En 1889 le libraire-éditeur grenoblois Alexandre Gratier publie ainsi une brochure de moins d'une cinquantaine de pages, Les patois comparés du département de l'Isère dans une fable de La Fontaine, le Loup et l'Agneau. Quarante-cinq cantons sur cinquante-trois sont représentés, grâce au concours de traducteurs locaux dont on ne sait d'ailleurs pas grand chose. Le département de l'Isère appartient pour l'essentiel à l'aire du francoprovençal, mais sa partie la plus méridionale est historiquement occitanophone, et Gratier fournit donc des versions de la fable dans une poignée de parlers de cette zone. Un peu plus tard, en 1890, le Bulletin de la Société dauphinoise d'Ethnologie publie une controverse entre un érudit local, le docteur Prompt, qui croit que ce que l'on parle en Oisans est fondamentalement du français, et le linguiste Devaux, qui considère, à bon droit, que l'Oisans est en fait traversé par la limite entre le francoprovençal et le « provençal »(Prompt1890). Les détails de ce combat (inégal!) nous importent ici moins que le fait que Prompt illustre son propos par une quinzaine de traductions de la fable le Loup et l'agneau dans la plupart des villages de l'Oisans. Mais dans un cas comme dans l'autre, on ne sort pas des marges iséroises.

De l'autre côté de la frontière, il y a le monumental recueil procuré en 1875 par Giovanni Papanti en hommage à Boccace: Sous le titre I parlari italiani in Certaldo alla festa del V centenario di Messer Giovanni Boccaccio (Livorno, Vigo), défilent quelque 700 traductions d'un petit conte du grand écrivain médiéval. Ces traductions fournies par des érudits locaux concernent aussi bien les diverses provinces du jeune royaume d'Italie, (disposées dans l'ordre alphabétique) que les «parlers italiens de populations ne faisant pas partie du royaume » : les «terres irrédentes » d'Istrie, de Dalmatie, et du « Tyrol italien », aux côtés de Saint-Marin et de la Suisse italophone, mais aussi... la Corse, Monaco, et le comté de Nice, laissé à la France une quinzaine d'années plus tôt. S'y ajoutent les langues étrangères parlées sur le sol italien : albanais, grec d'Italie du sud, allemand, slovène des Alpes, istro-roumain.... Les parlers de la Savoie, berceau de la dynastie, ont droit à leur chapitre à part, comme les idiomes «néo-latins», dont le « provençal « avec une version en « provenzale antico », du philologue Paul Meyer, et une version moderne, par Mistral. 
Dans cette masse de textes, il y a les deux provinces piémontaises de Turin et de Cuneo, dont la partie occidentale et montagnarde, déjà explorée par Biondelli, est de langue occitane, et francoprovençale pour le nord de celle de Turin, Aoste incluse. Contrairement à Biondelli, Papanti ne semble pas avoir trouvé de correspondant pour les vallées du Cuneese, mais pour la province de Turin, il fournit deux versions occitanes, (Mélezet, à côté de Bardonnechia, val Doire, Pramollo, bas Cluson), plus trois francoprovençales (Papanti 1875, 490-506).

Loccitan revient dans le chapitre consacré aux parlers « italiens » hors du royaume, avec Monaco, dont le parler ligurien voisine dans l'ouvrage avec celui de Menton, bien occitan, lui (Papanti 1875, 624-626). Le comté de Nice offre, outre une version en niçard, deux versions alpines. Au total donc, cinq textes occitans alpins.

Notre dernière collection est postérieure d'une dizaine d'années (1886). Il s'agit là encore d'une série de traductions d'un texte littéraire : le Salut à l'Occitanie de Fortuné Pin, imité de Florian, traduit en 107 idiomes (Montpellier, Hamelin, 1886) à l'occasion du centenaire de la mort d'un des grands écrivains d'oc du XVIIIe siècle, l'abbé Favre. Le maître d'œuvre de l'entreprise signe A. de Gagnaud, en fait le chevalier de Berluc-Pérussis, lié au monde des sociétés savantes du sud-est et au Félibrige : c'est un des confidents attitrés de Frédéric Mistral. Rien dans l'introduction qu'il donne à son recueil n'évoque l'œuvre de Papanti, mais on peut se demander s'il n'y a pas un rapport entre les deux. On note en effet un double parallélisme: une célébration centenaire ou pluricentenaire, Favre d'un côté, Boccace de l'autre, et le recours à deux textes littéraires comme support à un travail qui se veut aussi philologique. La différence : le choix de Berluc se porte sur un texte du fabuliste du XVIIIe, Florian, un Cévenol «monté » à Paris, paraphrasé par un élève de rhétorique des années 1820 qui se trouve être l'oncle de Berluc. Un double salut donc à deux ancêtres, l'un réel et propre au seul Berluc, l'autre virtuel et davantage inséré dans une généalogie félibréenne imaginaire -c'est le temps où les félibres de Paris adoptent pour leur fête annuelle la ville de Sceaux où est enterré Florian. Les contributeurs de l'entreprise, qui signent tous leur envoi, se recrutent soit dans la haute société félibréenne (les pères fondateurs, Mistral, Aubanel, Roumanille, ont apporté leur pierre comme d'autres félibres moins illustres mais néanmoins reconnus de leur temps) soit, pour les Hautes-Alpes notamment, parmi les élèves du lycée de Gap ou dans le clergé local.

La zone alpine est plutôt bien représentée, ne serait-ce que parce que Berluc, entre autres activités, figurait parmi les fondateurs de deux sociétés savantes départementales, celle des Hautes-Alpes, et celle des Basses-Alpes, département dans lequel il possédait d'ailleurs un domaine familial. Un des principes de classement de l'ouvrage est le critère provincial : dans le chapitre Provence figurent des spécimens des parlers du nord des Basses-Alpes. Curieusement, Berluc annexe à sa Provence un certain nombre de parlers des Hautes-Alpes, au motif, au demeurant assez acrobatique, que quoique considérés comme dauphinois, ils ont dépendu à un moment ou à un autre d'un comte de Provence. Mais d'autres parlers haut-alpins sont par contre rangés dans un chapitre Dauphiné avec Corps (Isère) et trois parlers drômois. Comme on voit, il n'est pas question de traverser la frontière : le Félibrige ne se souciait pas, alors, des "Provençaux » du Piémont, alors même que les travaux de Chabrand et de Rochas auraient pu l'y inciter.

Au total, ce sont donc des dizaines de spécimens des parlers alpins que l'on peut retrouver dans ces divers recueils. Si brefs soient-ils, ils n'en sont pas moins porteurs d'enseignements sur la situation linguistique d'une zone dialectale qui au XIXe siècle fournit assez peu d'auteurs occitans. Mais que nous apprennent-ils donc? 


\section{Ecrire des parlers}

Une première difficulté, technique, mais pas seulement : comment noter par écrit un « patois » laissé jusque là à l'oralité ? Pour ce qui est de l'occitan, les dernières traces des conventions graphiques élaborées au cours du Moyen Age ont disparu avec la fin du XVIe siècle. Depuis, ceux qui s'aventurent à écrire dans l'un ou l'autre des parlers d'oc en sont réduits à utiliser les conventions du français, dont l'ombre peut aller jusqu'à influencer le fond de la langue lui-même. La collection des paraboles du premier Empire témoigne de ces difficultés, mais elles ne disparaissent pas après Waterloo. Ce qui domine, c'est d'abord un certain nombre de constantes que l'on retrouve d'ailleurs dans les productions proprement littéraires du temps. C'est à la française que l'on note le phonème $/ \mathrm{u} /$, « ou ». D'où problème quand il s'agit de noter des diphtongues qui n'existent pas ou plus en français, mais subsistent en occitan : /'eu/, /'eu/, /'au/, /'ou/ ne peuvent être notés autrement que sous la forme éou, èou, aou, oou. Du moins les traducteurs postérieurs au Premier Empire évitent-ils de parsemer leur version de ces K qui ornent alors les textes venus d'Embrun ou du Queyras. Mais ils éprouvent parfois la même difficulté à couper correctement les mots. Le docteur Prompt propose en 1894 une traduction du Loup et l'agneau dans le parler de Besse, dans la partie occitanophone de l'Oisans :

Ur agnel se disalteravo

Diens le courant dur aigo puro

Ugn loup arrivo a zeugn que tsertsavo aventuro

Et que la famm en di que lous leuqs attiravo ».(Prompt 1890, 98)

Le traducteur essaie du mieux qu'il peut de noter la prononciation de son parler, en redoublant les consonnes finales (fortt, d'abortt, vouss... et loupp plus loin dans la fable) pour bien indiquer qu'elles se conservent et se prononcent (ce n'est pas le cas dans la partie francoprovençale de l'Oisans). Mais par contre, il a du mal à interpréter correctement ce « dur aigo puro » qu'il aurait pu noter « d'ur'aigo puro»: il s'agit de l'article indéfini avec le traitement spécifique à l'Oisans et au briançonnais le rhotacisme du -n- intervocalique. Quant à «di que lous », il s'agit en fait du démonstratif (d') iquelous, correspondant à la forme ordinaire en occitan aqueles/aquelos (l'influence du francoprovençal voisin sefaisant sentir, là encore comme dans une bonne partie du briançonnais et des vallées vaudoises : « iquaou pais ilai », ce pays-là, dit la version du Monêtier fournie par Chabrand-de Rochas).

De l'autre côté de la frontière, les traducteurs se posent le même problème : comment noter leur parler avec les conventions de la langue écrite usuelle chez eux. Sauf qu'ils disposent de deux modèles. Dans les vallées du nord, qui ont relevé du Dauphiné français jusqu'en 1713, et a fortiori dans les vallées vaudoises où le français est la langue du culte protestant, le modèle est français : "un homme avie doû bos : le plu jouve de ilou dit à soun paire ", trouve-t-on dans la version d'Oulx publiée par Chabrand-de Rochas. Ce qui correspond sous la plume du pasteur Pierre Bert pour la Val Pellice à « Un hom avia dui fill; é lou pi giouve di à so pare » où l'on note (outre l'ombre du piémontais qui fait remplacer soun paire par so pare) l'introduction d'un graphème étranger au système français, et renvoyant à un modèle transalpin : le gi- de giouvou (Chabrand de Rochas 1877, 152-154).

Transalpin, pas forcément italien standard : il existe une tradition écrite du piémontais 
qui peut servir aux transcripteurs de la région où elle est en usage. Mais de toute façon, l'adaptation de l'une ou l'autre à l'occitan ne se fait pas si simplement. Il y a le problème du /y/, que le piémontais connaît, mais non l'italien. Le parti pris par Biondelli et ses collaborateurs (Biondelli 1853, 505 sqq.) écrivant pour un public plus large que celui de la province, est de noter ce phonème par ü. La version de Fenestrelle, haut Cluson, commence donc ainsi : « ün ome avio du garsuns; e le plü giuve a dit». Ce qui correspond pour Oncino (Val po) à « ün om a l'a agü dui figl; lu pü giove a l'a dič a son pare », d'où problème : non point pour la notation de /y/ (ü) mais pour celle de /u/: si pour « dui » et «lu », l'article défini, le traducteur choisit une notation à l'italienne, il n'en va pas de même pour « giove » et « son »qu'il conviendrait de noter "giuve, sun », comme à Fenestrelle -un siècle plus tard, c'est bien un /u/ que Hirsch (Hirsch 1978, 47) a noté à Oncino (dzuve) dans une version nouvelle de...la parabole. C'est qu'en piémontais, le /u/ peut justement se noter par «o ». Dans une bonne partie des versions des vallées de la province de Cuneo, c'est ce « giove » que l'on retrouve, d'où l'erreur du brave Biondelli, qui oppose ainsi, sur la foi des traductions dont il dispose, les parlers du sud à des parlers du nord qui, dit-il, prononcent / $\mathrm{u} /$ comme les parlers occitans de l'autre versant (Biondelli 1853, 479).

D’une manière générale, le modèle italien joue sur la notation de la latérale palatalisée «figl », « viegl » (vieux) à Oncin. Il joue aussi dans le cas de «che», « achel », « achest ». En revanche, les traducteurs sont très gênés par les affriquées finales: comment noter les dérivés de FACTU, DICTU ? Côté français, c'est somme toute facile : on aura, selon les versions, dich fach, ditch fatch, ou ditz fatz. Sur l'autre versant, Biondelli en est réduit à utiliser un c muni d'un signe diacritique, le circonflexe renversé : dič, fač, qu'il emprunte, nous dit-il dans son introduction, aux langues slaves.

Le plus embarrassé des traducteurs mobilisés par Biondelli a sans doute été ce pasteur Bert qui fournira un peu plus tard la version vaudoise de Chabrand-de Rochas. La version qu'il publie en 1853 est rigoureusement identique, sur le fond, à celle de 1877. Mais la graphie n'est pas du tout la même:

Ün om avia düi fil ; e lu pi giouvou di a so pare...

Ni o, ni u : ou comme sur le versant français (cf supra).

Plus loin, là où en 1877 on trouvera:

" apreu qu'a l'ha agù tut despendù, una gran famina é vengùa ente quel païs lai » on trouve en 1853 :

« aprö ch'a la agü tüt despendü, üna gran famina è vengüa ente quel pai lai »

Où l'on voit appliquer la règle graphique italienne et piémontaise dans che, alors que pour quel, c'est la notation à la française qui refait surface, là où on attendrait logiquement une forme de type chel...

Une vingtaine d'années plus tard, les contributeurs occitans à l'ouvrage de Papanti rencontrent à peu près les mêmes difficultés. Ils les résolvent de façon diverse. La version du Mélezet, suit les règles graphiques d'un français qui à ce stade est encore la langue de l'école dans sa vallée: 
A disou doun que do tein do premier rei de Cipre, après que Godefroi de Bullioun s'ère rendu mêtre de la Terre Seinte, l'è arriva qu'une femme noble (ovvero, de noublesse) de la Gascougne è anà in pelerinage o Seint Sepulcre.

Par contre, quelques pages plus loin, la version de Pramollo annonce dans une note «L'ortografia è italiana». Ceci étant, elle note le e muet à la piémontaise (ë) et signale que «l'u a il suono dell'u lombardo » c'est-à-dire que c'est un /y/. Du coup, pour noter le /u/, il faut recourir à la solution piémontaise : o. Ce qui donne :

Doncre v'lo dio, che'nt ji temp dël prim rei d'Cipri, apre che Gotifrè de Bullion avè aquistà la Tera Santa, j'est arrivà che na sgnora de Gascogna, il j'est anà n'pelegrinagi al Sepolcre.

Même choix italianisant à Menton. Quoique...

Digo dounca, che nu tempe d'ou primo Re de Cipri, aprèss a conquista che Gottifré de Bouglione a fatch de ra terra Santa, ez arribà che una frema nobila de Gascogna ania (sic pour avia ?) anà aou Sepulcro en pellegrinage.

On voit bien que se mêlent ici graphèmes transalpins (che), et " français » : " ou », " (che dee pronunciarsi come in francese » précise une note) et " aou » On note aussi fatch, en remarquant toutefois que quelques lignes plus loin la même affriquée est notée à l'italienne: facce (pp masculin avec voyelle d'appui), ou staccia (ppf du verbe être).

En comté de Nice, c'est le modèle italique qui domine clairement (et qui dominera encore une bonne partie de l'écrit d'oc du comté) :

Bollène : l'ortografia è italiana; tutta via l'ü si pronunzia come l'u francese :

Diu adunco che ai temps dal prim Rej de Cipri, après che Giufrei d'Bugliun aghè conquistat Terro Santo, arivè che uno belo fremo d'Gascogno se n'anè en pelegrinage au Sant sepulcre (Papanti 1875,496 et 500).

En revanche, pour noter l'affriquée, le traducteur a recours à une solution personnelle : faç, faça. On voit aussi qu'au mépris de ses propres consignes, il note uno sans tréma...

Sospello : si è seguita l'ortografia italiana, salvo pei suoni estranei alla lingua. Ce qui donne:

Dunch digu, chë as temps d'u premier Rëi dë Cipro, apres fac a cunquista d'a tera santa da Gufredo da Buiùn, ës capità chë üna gran signura dë Gascogna anè ën pëlëgrinage au Sepülcrë.

Le ë note, comme en piémontais, le le/ neutre. Pour l'affriquée, sont choisies les solutions fac, sans signe diacritique, et facia.

Dans tous les cas, le choix de la graphie ne renvoie pas seulement à des impératifs d'ordre technique (comment reproduire au mieux les sons d'un parler pour ceux qui ne l'ont jamais entendu) mais aussi, sinon surtout, à des traditions culturelles, voire à des questions d'identification nationale: d'où le dilemne des Occitans des vallées du nord, écartelés entre 
leur appartenance à l'Italie unifiée et le souvenir du temps où pour des raisons politiques (Val Doire) ou religieuses (vallées vaudoises) leur référent écrit était le français. D’où aussi la position des invités niçois de Papanti, qui choisissent la référence à l'italien pas seulement parce qu'au cours du XIXe siècle et jusqu'à l'annexion par la France c'était la langue de l'éducation chez eux, mais encore parce qu'après 1870, la cause d'un certain irrédentisme rencontre un succès relatif dans une partie des élites niçoises.

Avec la brochure publiée par Berluc-Pérussis, félibre éminent, chez l'éditeur montpelliérain Hamelin, lié de près au Félibrige comme aux romanistes de la Société pour l'Etude des langues romanes, on pourrait penser que la question de la graphie de l'occitan et de sa normalisation va être réglée une bonne fois pour toutes. C'est vrai pour l'essentiel, et les solutions félibréennes, notamment en ce qui concerne la notation des diphtongues au, èu, éu ... sont majoritaires, y compris dans la notation de parlers assez différents du provençal mistralien. Mais tous les contributeurs ne sont pas félibres. Ils ont parfois l'excuse de devoir noter des parlers vraiment divergents par rapport au modèle provençal. Le traducteur chargé du parler de Corps doit ainsi rendre compte d'une caractéristique de ce parler : la chute des -s devant consonne et à la fin des mots peut entraîner un allongement compensatoire de la voyelle précédente. Il écrira donc : pertout entendou ta louanzâ (j'entends partout tes louanges ) ou Eei per tu que lou Roumain meeipreseroun aquéu bèu paï de l'Auzounia : notre homme adopte la règle félibréenne pour les diphtongues, mais procède à ses propres inventions pour rendre compte des conséquences de la chute du -s. (Salut...1886, LIX).

Le contributeur de Luc en Dois, un prêtre lui aussi, a ses propres solutions :Te saluoue, ou belle oucitanieo, terro touzours aima dos cioux. Cioux comme cieux, de même que les troupeaux ne peuvent s'écrire autrement dans son parler que sous la forme troupéoux. Et il propose des formes de passé simple du style negligerouent, ferouent, où les trois dernières lettres ne semblent avoir d'autre fonction que de rappeler les formes du français, là où d'autres notent sans malice ce qui se prononce, (fèroun ou faguèroun) (Salut... 1886, 56). Chabrand, le coauteur du travail sur les parlers des Alpes Cottiennes, participe à l'entreprise de Berluc, mais sans renoncer à sa pratique antérieure : il écrira donc aoutres, déou, et non autres ou déu.

Et nos traducteurs ont parfois du mal à interpréter correctement les formes qu'ils notent: Chabrand et l'élève du lycée de Gap chargé du parler d'Arvieux sont confrontés à la forme interrogative particulière au queyrassin et aux parlers voisins des hautes vallées piémontaises, caractérisés par l'emploi obligatoire du pronom personnel sujet : " que diraije » se traduit donc par que direi qu'io (Arvieux) ou que direi qu'iou (Chabrand). La traduction d'Arvieux fournit une piste susceptible d'expliquer cette forme curieuse : " a te saluouc », je te salue, avec donc ce -c final que l'on retrouve déjà à la première personne des verbes aussi bien dans les textes vaudois que dans les mystères briançonnais du XVe siècle : en bonne logique c'est donc que direic-ieu qu'il aurait convenu d'écrire.

\section{Parler des parlers}

Il y a donc des traductions, qui permettent à l'observateur de se faire une idée de ce qu'est le paysage linguistique des Alpes occitanes. Mais comment aller au delà de la simple lecture de ces spécimens de langue ? Comment lire, au delà des textes, le paysage qu'ils dessinent ? Ce n'est pas a priori le travail demandé aux traducteurs par les donneurs d'ordre. Et de fait, que ce soit dans l'enquête du Premier Empire ou dans celles qui vont suivre, des 
deux côtés de la frontière, beaucoup, pour ne pas dire la plupart, se bornent à traduire le texte support qui leur est proposé. Peu s'aventurent à donner les clés du système graphique qu'ils emploient : on a vu des exemples dans la collecte de Papanti. Plus rares encore ceux qui se sont risqués à prendre le recul nécessaire à un commentaire des données qu'ils fournissaient. Ceux-là peuvent être les commanditaires de la collecte. Eugène Coquebert de Montbret ouvre ses Mélanges sur une longue étude de la situation linguistique que son enquête lui a révélée, et donne l'ébauche d'une cartographie, voire d'une statistique des langues parlées sur le sol de l'Empire. Il propose ainsi une limite entre oc et oïl, globalement exacte pour la partie occidentale, plus vague vers l'est -il ne distingue pas le francoprovençal de son voisin occitan, confusion qui perdurera même après l'identification du premier par Ascoli. Par ailleurs, un certain nombre de ses correspondants, soit au moment de l'enquête elle-même, soit dans les ouvrages qu'ils publient par la suite, produisent des analyses du corpus qu'ils ont rassemblé. René Merle a étudié ces analyses, et je renvoie à ses travaux (Merle 1990, 476-502).

Bien des années plus tard, Chabrand et de Rochas proposeront pour leur part, une frontière linguistique entre Oc et Oỉ, qui suit le cours de l'Isère à partir du Rhône, puis celle de la Bourne, laissant à l'Oill Villard de Lans, rangé aujourd'hui côté occitan, avant de passer au nord du Trièves le long du Drac, et d'englober une partie de l'Oisans, pas autrement précisée. Ce n'est somme toute pas si mal vu, et rompt avec l'attribution ordinaire du francoprovençal à l'occitan. Plus à l'est, la langue d'oc englobe Bardonnèche et les vallées vaudoises. Les auteurs ne se prononcent pas sur l'appartenance des vallées situées plus au sud. En revanche, ils régalent le lecteur de considérations de nature esthétique sur les divers « patois » des Alpes Cottiennes : celui du Queyras, est «franc et sonore », celui de la Vallouise est «lent et semé d'exclamations », vers Briancon par contre il est " pesant et dur comme celui de la Savoie » voisine, etc. (Chabrand de Rochas 1877, 2-7). Ce qui rappelle la prose des traducteurs du Premier Empire.

Pour nos deux érudits cottiens, l'appartenance des Alpes du sud à l'aire d'oc ne fait donc pas débat. C'est un point de vue au reste assez largement partagé, dès le Premier Empire, même si alors prévaut l'idée de «patois » qui ne sont que mélange plus ou moins bien dosé de langues véritables, ou simples déformations du seul français, idée tout au plus corrigée parfois par celle qu'il existe malgré tout une entité " provençale » qui a sa propre autonomie. Plusieurs années plus tard, la redécouverte des Troubadours par Raynouard aidant, l'autonomie du provençal, alias langue romane, finit par s'imposer.

Côté « italien », il y a peu à trouver chez Papanti. Les correspondants de Menton et du Comté de Nice se bornent aux indications sur la graphie que nous avons déjà citées. Quant à Biondelli, c'est lui-même qui se charge de décrire les parlers gallo-italiques dont il publie des spécimens. Dans son Piémont qu'il divise en trois aires -piemontese, canavese et monferrino, c'est dans le piemontese qu'il range les parlers « alpigiani », occitan et francoprovençal mêlés. Mais les deux variétés n'ont pas le même statut. Les trois textes francoprovençaux n'ont pas droit à une dénomination particulière, et sont simplement caractérisés comme rozzi e informe, grossiers et informes. Plus au sud, ce que voit Biondelli, ce sont des parlers certes piémontais, mais qui annoncent par certains traits les parlers occitanici de l'autre versant. Il précise les traits qui lui paraissent justifier cette idée : l'évolution PATRE>paire, la palatalisation de CA/GA (ciantar), le pluriel sigmatique, en tout cas pour les parlers les plus septentrionaux (lus cusciuns, les cochons), un trait syntaxique « se levar» face à des parlers piémontais qui pratiquent la 
postposition du pronom réfléchi, un certain nombre de traits de lexique, aussi. Ce n'est pas si mal vu dans l'ensemble, là encore.

«Occitans» ou piémontais, alors, ces parlers des montagnes ? Pour Biondelli il y a moins limite franche que transition progressive, leggeri e quasi impercettibili gradazioni; et, un peu plus loin :

Di mano in mano che c'inoltriamo su per l'erto dei monti, il dialetto piemontese, trasformandosi assume alquante forme dei dialetti occitanici, cio che porge nuovo interesse al linguista que nell'incorretta favella dell'alpigiano scopre ancor vive le vestigia della lingua dei trovatori. (à mesure que nous nous avançons sur les pentes des montagnes, le dialecte piémontais, en se transformant, prend certaines formes des dialectes occitans, ce qui fournit un intérêt nouveau au linguiste qui dans le parler incorrect de l'Alpin découvre, vivants encore, les vestiges de la langue des Troubadours) (Biondelli 1853, 475)

Quant au parler de Fenestrelle, pour lui, " plutôt qu'un dialecte italien, il semble un dialecte français travesti à l'italienne »(Biondelli 1853, 485).

Au delà de l'emploi précoce du vocable « occitan » pratiquement inutilisé en France à cette date (l'Occitanie de Florian et Berluc, c'est le Languedoc), on constate qu'on est déjà dans la problématique de la tapisserie, cette métaphore qui aura son moment de succès chez les dialectologues, français comme italiens, attachés à célébrer l'unité linguistique de leur patrie (Brun-Trigaud 1990, Martel 2009, 209-239). Signe sans doute, chez Biondelli, d'un certain embarras patriotique : peut-on si facilement que cela assigner ces parlers à une appartenance étrangère? Leur présentation comme parlers piémontais touchés par des influences transalpines permet de concilier réalité observable, et respect d'une frontière politique qui même avant l'unification à venir prochainement est déjà de rigueur en Italie.

Au moment où paraît le Salut à l'Occitanie, le débat sur la question de l'existence ou non de limites entre langue d'oc et langue d'oïl est déjà bien engagé, entre d'un côté Paul Meyer et Gaston Paris, et de l'autre les félibres montpelliérains Tourtoulon et Bringuier, ceux qui ont parcouru et balisé sur une partie de son parcours la frontière dont les Parisiens nient l'existence. Dans son introduction, Gagnaud-Berluc cite ses deux confrères félibres et leur travail, et expose ses propres idées sur la partition dialectale :

«On sait que parmi les caractères qui distinguent les idiomes entre eux, il n'en est pas deux qui aient absolument le même périmètre géographique. Il faut donc de nécessité absolue pour tracer sur la carte la ligne divisoire des dialectes, s'attacher à un caractère unique, le plus saillant. C'est dans le mot par excellence, le verbe, que l'on doit naturellement chercher ce signe dominateur qui se retrouvera presque fatalement dans chaque phrase » (Salut... 1886, 11).

On reconnaît au début de la citation l'argument massue des Parisiens, sur l'impossibilité de dessiner des limites à partir de phénomènes phonétiques dont les aires ne coïncident pas. Mais Berluc, non sans insolence, choisit de ne prendre cet argument en compte que pour mieux le contourner, en délaissant les critères phonétiques et phonologiques au profit du critère de la morphologie verbale. C'est sur cette base qu'il divise les parlers provençaux en trois zones, celles d'Amou (Alpes), d'Ame (Bas-Rhône) et d'Ami (Provence centrale et orientale), en fonction du morphème de première personne du singulier du verbe aimer. Il 
y a donc non seulement une langue d'oc, mais aussi en son sein des dialectes identifiables. C'est prendre position vigoureusement dans le débat. Mais c'est que la visée de la brochure est au moins autant scientifique que militante: son introduction s'ouvre sur une profession de foi décentralisatrice et anti-parisienne, avant de se clore sur une profession de foi félibréenne et renaissantiste, autour de la question de l'orthographe mistralienne, seule susceptible de conférer à la langue, dans sa diversité, la dignité nécessaire à une grande langue de culture. A la suite, tout en acceptant l'usage du dialecte pour la littérature populaire, c'est la langue de Mistral qui lui semble seule convenir pour les lettrés..

Mais le premier âge de la dialectologie s'achève. Quatre ans plus tard, en 1890, Giuseppe Morosi note les paraboles traduites dans les parlers vaudois qu'il étudie dans un système phonétique original, celui de l'Archivio Glottologico Italiano. Encore quelques années, et c'est avec l'alphabet Rousselot que les universitaires français noteront les "patois » qu'ils étudieront. Les considérations esthétiques et militantes (en apparence du moins) ne seront plus de saison...

Faut-il pour autant rejeter comme obsolètes et sans intérêt pour le chercheur d'aujourd'hui les paraboles, fables, contes et autres petits textes martyrs dont le XIXe siècle a fait l'usage qu'on a vu? Tels quels, avec leur graphie parfois difficilement interprétable, leur littéralisme aussi, générateur de calques du français, ces textes sont riches de renseignements. On l'a dit, pour certains parlers, il s'agit bel et bien de la première et parfois de la seule trace écrite qu'ils aient laissée. La photo est tremblée, mais c'est la photo d'un parler, et à ce titre on peut la comparer à ce que sont devenus, depuis, les parlers considérés. On peut ainsi constater les progrès vers le nord de l'article pluriel neutralisé provençal « lei » aux dépens de la forme occitane traditionnelle "los/las», conservée dans les montagnes : en 1886 le traducteur de Digne-Sieyès note que lous et las ne sont plus employés que par les vieillards. Et même entre deux états d'un même parler au XIXe siècle, on entrevoit les évolutions en cours : la version de 1812 de celui de Barcelonnette (nord des Basses-Alpes) employait massivement une forme de passé simple. Chez Chabrand et de Rochas, le spécimen fourni pour la même vallée ne mentionne plus ces formes qu'entre parenthèses : le temps devenu normal, c'est le passé composé qui a seul survécu au siècle suivant. On voit que ces vieux documents peuvent être instructifs.

Et ils le sont tout autant si ce n'est plus dès qu'il s'agit de voir comment ces hommes qui se chargent de noter les " patois» de leur lieu les considèrent, dans quelle mesure ils arrivent à dépasser le local, justement, pour resituer leur parler dans un ensemble autrement plus vaste. On mesure de la même façon l'impact sur ce regard des progrès de la romanistique au fil du XIXe siècle. On entrevoit même, fugitivement, l'ombre des débats qui traversent alors cette romanistique dès lors que l'idéologique (le fait national en l'occurrence) interfère avec le scientifique. Autant de choses que l'on sait, bien sûr, et de longue date, mais que le détour par ces collections de traductions plus ou moins adroites dans la parlure des gens de peu de textes de registre élevé par des lettrés de plus ou moins haut vol permet peut-être de voir sous un autre angle. 


\section{Bibliographie}

Salut...1886, Salut à l'Occitanie de Fortuné Pin, imité de Florian, traduit en 107 idiomes [BerlucPerussis, Léon de,] (Montpellier, Hamelin)

Brun-Trigaud, Gyslaine 1990, Le Croissant, le concept et le mot, Lyon, Université Lyon 3.

Chabrand, J. A., de Rochas, A, 1877, Le patois des Alpes Cottiennes, Grenoble ; sn.

Courouau, Jean-François, 2011, «La naissance d'un savoir entre Aufklärung et romantisme » in Savoirs romantiques, une naissance de l'ethnologie, dir. Daniel Fabre, Nancy, Presses universitaires, pp. 79-101.

Hirsch, Ernst, 1978, Provenzalische Mundarttexte aus Piemont, Tubingen, Niemeyer.

Martel, Philippe, 2009, «La tapisserie volante : autour de la « questione ladina » in C. Alen Garabato, T. Arnavielle. C. Camps, La Romanistique dans tous ses états, Paris, l'Harmattan, pp. 209-239.

Merle, René, 1990, Lécriture du provençal de 1775 à 1840, Béziers, CIDO.

Morosi, Giuseppe, 1890 «Lodierno linguaggio dei Valdesi del Piemonte », Archivio Glottologico Italiano XI, 1890, pp. 309-415 ; XII, 1890/92, pp. 28-32.

Prompt, Dr, 1890, «Etude sur la langue de l'Oisans », Bulletin de la Société Dauphinoise d'Ethnologie, pp. 89-99 (les fables occupent les pp. 96-99). Réponse de Devaux pp. 173-179., suivie de la réponse de Prompt (pp. 180-187).

Papanti, Giovanni,1875, I parlari italiani in Certaldo, Livorno, Vigo. 